\title{
Accuracy of Contrast Extravasation on Computed Tomography for Diagnosing Severe Pelvic Hemorrhage in Pelvic Trauma Patients: A Meta-Analysis
}

\author{
Sung Nam Moon $1,+\left(\mathbb{C}\right.$, Jung-Soo Pyo ${ }^{2,+}$ and Wu Seong Kang $3, *(\mathbb{C}$ \\ 1 Department of Radiology, Wonkwang University Hospital, Iksan 54538, Korea; sungnam0422@gmail.com \\ 2 Department of Pathology, Eulji University School of Medicine, Uijeongbu Eulji University, \\ Kyeonggi-do 11759, Korea; anapyojs@gmail.com \\ 3 Department of Trauma Surgery, Wonkwang University School of Medicine, Wonkwang University Hospital, \\ Iksan 54538, Korea \\ * Correspondence: wuseongkang@naver.com \\ + These authors contributed equally to this work.
}

Citation: Moon, S.N.; Pyo, J.-S.; Kang, W.S. Accuracy of Contrast Extravasation on Computed Tomography for Diagnosing Severe Pelvic Hemorrhage in Pelvic Trauma Patients: A Meta-Analysis. Medicina 2021, 57, 63. https://doi.org/ 10.3390/medicina57010063

Received: 20 December 2020 Accepted: 10 January 2021 Published: 12 January 2021

Publisher's Note: MDPI stays neutral with regard to jurisdictional clai$\mathrm{ms}$ in published maps and institutional affiliations.

Copyright: (C) 2021 by the authors. Licensee MDPI, Basel, Switzerland. This article is an open access article distributed under the terms and conditions of the Creative Commons Attribution (CC BY) license (https:// creativecommons.org/licenses/by/ $4.0 /)$.

\begin{abstract}
Background and objective: The early detection of underlying hemorrhage of pelvic trauma has been a critical issue. The aim of this study was to systematically determine the diagnostic accuracy of computed tomography (CT) for detecting severe pelvic hemorrhage. Materials and Methods: Relevant articles were obtained by searching PubMed, EMBASE, and Cochrane databases through 28 November 2020. Diagnostic test accuracy results were reviewed to obtain the sensitivity, specificity, diagnostic odds ratio, and summary receiver operating characteristic curve of CT for the diagnosis in pelvic trauma patients. The positive finding on CT was defined as the contrast extravasation. As the reference standard, severe pelvic hemorrhage was defined as an identification of bleeding at angiography or by direct inspection using laparotomy that required hemostasis by angioembolization or surgery. A subgroup analysis was performed according to the CT modality that is divided by the number of detector rows. Result: Thirteen eligible studies ( 29 subsets) were included in the present meta-analysis. Pooled sensitivity of CT was 0.786 [95\% confidence interval (CI), 0.574-0.909], and pooled specificity was 0.944 (95\% CI, 0.900-0.970). Pooled sensitivity of the 1-4 detector row group and 16-64 detector row group was 0.487 (95\% CI, 0.215-0.767) and 0.915 (95\% CI, 0.848-0.953), respectively. Pooled specificity of the 1-4 and 16-64 detector row groups was 0.956 (95\% CI, 0.876-0.985) and 0.906 (95\% CI, 0.828-0.951), respectively. Conclusion: Multi-detector CT with 16 or more detector rows has acceptable high sensitivity and specificity. Extravasation on CT indicates severe hemorrhage in patients with pelvic trauma.
\end{abstract}

Keywords: trauma; computed tomography; hemorrhage; angiography; pelvis

\section{Introduction}

Pelvic fracture accompanying hemorrhage has been a large challenge for clinicians. Especially in hemodynamic instability due to pelvic ring injuries, mortality rates remain high (up to 35.7\%) [1-3]. Bleeding is the most influential factor on the severity of pelvic injury. Therefore, early detection of underlying hemorrhage of pelvic trauma has been a critical issue. Despite well-experienced trauma surgeons, the surgeon's gestalt is not sufficient to detect signs of catastrophe [4]. The previous prediction model for severe hemorrhage demonstrated predictors including mechanism of injury, systolic blood pressure, heart rate, hemoglobin, lactate, and focused abdominal sonography for trauma (FAST) [5]. However, there were no high-quality, evidence-based models. Contrast-enhanced computed tomography (CT) is a valuable diagnostic tool that reveals bleeding using contrast blush. Nevertheless, the role of CT is limited in hemodynamically unstable patients, because it can be a time-consuming process and delay resuscitation. However, both CT scanning and 
intervention are available simultaneously due to the recent revolutionary development of the hybrid-ER system and angio-CT suite in several trauma centers [6,7]. Moreover, since multi-detector CT has evolved, the diagnostic accuracy of CT has improved remarkably. Therefore, we conducted this systematic review and meta-analysis to determine the diagnostic test accuracy of CT for detecting severe pelvic hemorrhage. In contrast to the recent meta-analysis [8] that showed high sensitivity and specificity in 64-detector row CT, we intended to investigate if 16 or higher detector row CT might have a sufficient diagnostic accuracy.

\section{Materials and Methods}

\subsection{Published Study Search and Selection Criteria}

This study was performed in accordance with the Preferred Reporting Items for Systematic Reviews and Meta-Analysis of Diagnostic Test Accuracy (PRISMA-DTA) statement [9]. Relevant articles were obtained by searching PubMed, EMBASE, and Cochrane databases through 28 November 2020. These databases were searched using the following keywords: “(pelvic OR (pelvic fracture) OR (pelvic bone fracture)) AND (computed tomography) AND ((contrast extravasation) OR (arterial extravasation) OR (contrast blush) OR extravasation))." In addition, we manually searched the reference lists of relevant articles. The titles and abstracts of all searched articles were screened for exclusion. Review articles and previous meta-analyses were also screened to obtain additional eligible studies. Search results were then reviewed, and articles were included if the study investigated the diagnostic accuracy of pelvic CT.

The inclusion criteria for diagnostic test accuracy (DTA) reviews were the following: (1) the study population included pelvic trauma patients; (2) as an index test, contrastenhanced CT was performed. The positive finding on CT was defined as contrast extravasation; (3) as the reference standard, severe pelvic hemorrhage was defined as an identification of bleeding at angiography or by direct inspection using laparotomy that required hemostasis by angioembolization or surgery; (4) the purpose of the study was to evaluate the diagnostic accuracy of CT in pelvic trauma patients; (5) adequate information was provided to build a $2 \times 2$ table consisting of true positive (TP), false positive (FP), false negative (FN), and true negative (TN). Those articles that studied another disease, nonoriginal articles, non-human study, pediatric study, or non-English-language publications were excluded.

\subsection{Data Extraction}

Data from all eligible studies were extracted by two investigators. Extracted data from each of the eligible studies included [10-22] the first author's name, year of publication, study location, design, and period; the number of patients analyzed, type of rows of detector elements ( 1 to 64 detector row), and time from admission to angiography. In addition, the number of TP, FP, FN, and TN for contrast extravasation in diagnosing severe pelvic hemorrhage were collected.

\subsection{Quality Assessment}

All studies were independently reviewed by two investigators. Any disagreement concerning the study selection and data extraction were resolved by consensus. As recommended by the Cochrane Collaboration, to evaluate the risk of bias in DTA, the Quality Assessment of Diagnostic Accuracy Studies (QUADAS)-2 tool was used [23]. Disagreements were resolved by discussion with the third independent author. The QUADAS-2 assesses four domains for bias and applicability as follows: (1) patient selection: risk of bias is considered high if there is no consecutive patient enrollment and avoidance of case-control design or inappropriate exclusion; (2) index test: risk of bias is considered high if the index test results were interpreted without blinding to the reference standard; (3) reference standard: risk of bias is considered high if the reference standard could classify the target condition incorrectly; (4) flow and timing: risk of bias is considered high if not all 
patients were included with the same criteria or if there was an inappropriate time interval between the index test and reference standard.

\subsection{Statistical Analysis}

We investigated the contrast extravasation according to clinical characteristics from eligible studies and computed the point estimate to combine single descriptive statistics [24]. As the eligible studies used populations with heterogeneity, a random-effects model was more appropriate than a fixed-effects model. Heterogeneity between eligible studies was checked using probability statistics ( $p$-value). To evaluate publication bias, Begg's funnel plot and Egger's test were conducted. If significant publication bias was found, the fail-safe $\mathrm{N}$ and trim-fill tests were also conducted to confirm the degree of publication bias. We conducted a bivariate diagnostic random effect meta-analysis that considers the joint distribution of sensitivity and specificity, allowing for a cross-study correlation. We calculated the pooled sensitivity, specificity, and diagnostic odds ratios (DOR) according to individual data collected from each eligible study in the various categories of comparison. As data were heterogeneous, accuracy data were pooled by fitting the summary receiver operating characteristics (SROC) curve (bivariate model) and measuring the area under the curve (AUC). An AUC close to 1 and 0.5 indicated a strong and poor test, respectively. Results with $p$-values $<0.05$ were considered statistically significant. Besides, we conducted additional meta-regression analysis by the location of eligible studies to find the possible cause of heterogeneity. All statistical analyses were conducted using a Comprehensive Meta-Analysis software package (Biostat, Englewood, NJ, USA) and R software (The R Foundation for Statistical Computing, version 3.6.0). In addition to base package of $R$, mada (version 0.5.8) and meta (version 4.10-0) packages were used.

\section{Results}

\subsection{Selection and Characteristics}

A total of 375 studies were identified through database searching. Among the searched studies, 247 were excluded from title and abstract review because they were non-original $(n=69)$, studied other diseases $(n=116)$, non-human studies $(n=1)$, or were written in a non-English language $(n=13)$. After full-text review, 28 were excluded because they were insufficient data $(n=26)$, non-original article $(n=1)$, or pediatric study $(n=1)$. Finally, 13 studies (29 subsets) comprising 2642 patients were included in the present meta-analysis and DTA review (Figure 1), and detailed information about the eligible studies is shown in Table 1. To analyze for overall patients and subgroups, each study was investigated dividing into three subsets, such as overall, contrast extravasation, and no contrast extravasation. 
375 studies identified through database searching

Pubmed $(n=196)$, EMBASE $(n=177)$, Cochrane $(n=2)$

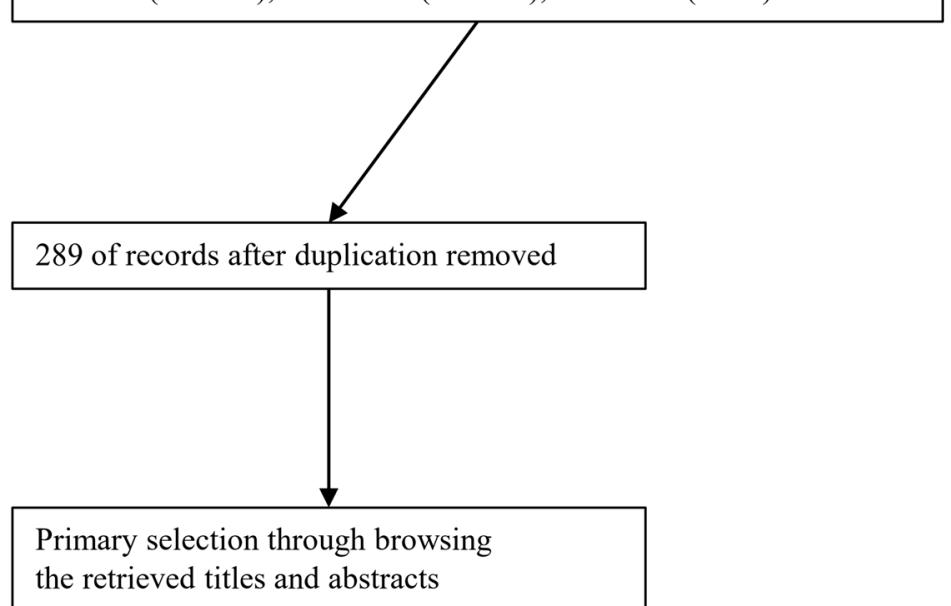

the retrieved titles and abstracts

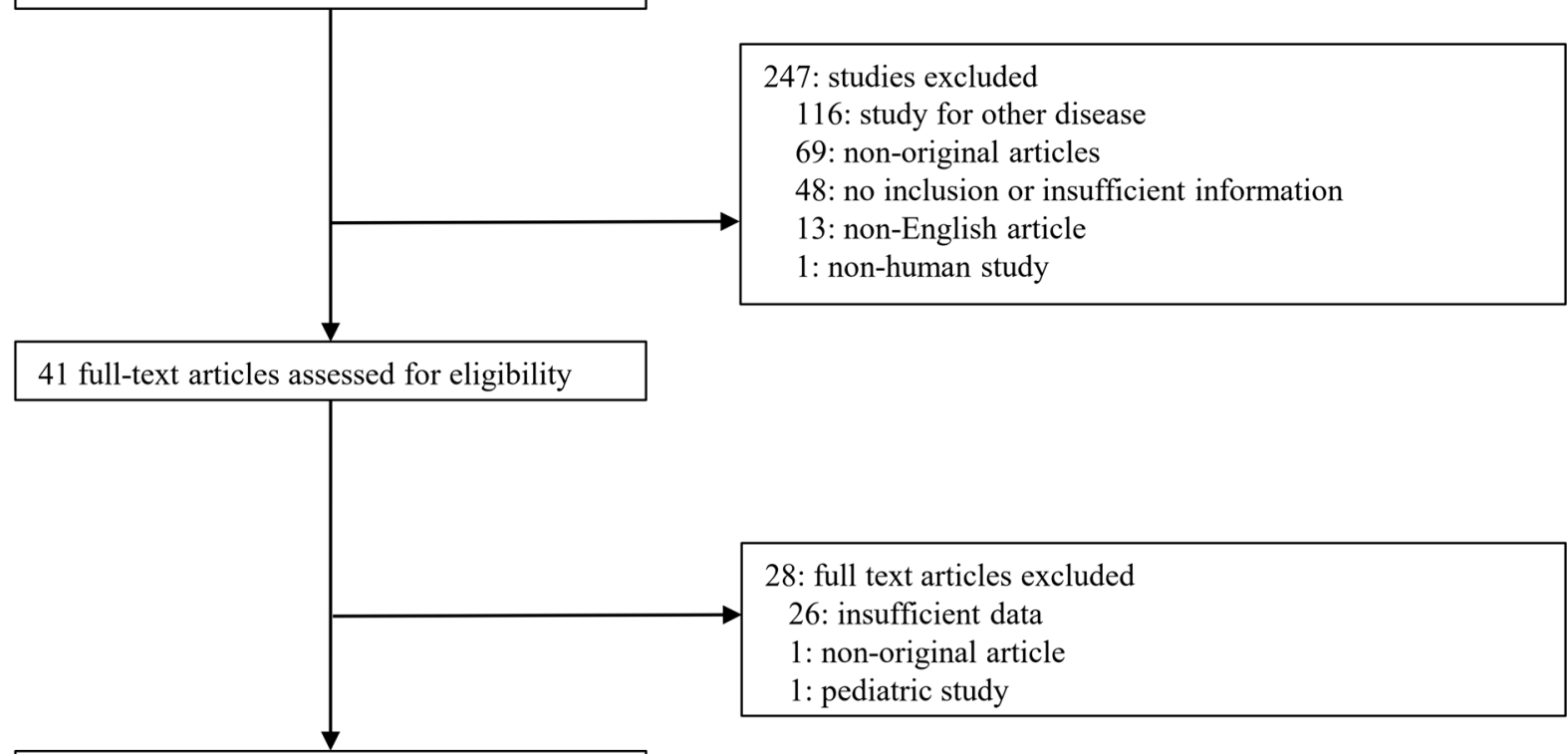

13 studies included in the meta-analysis

Figure 1. Flowchart summarizes literature and study selection. 
Table 1. Main characteristics of the eligible studies.

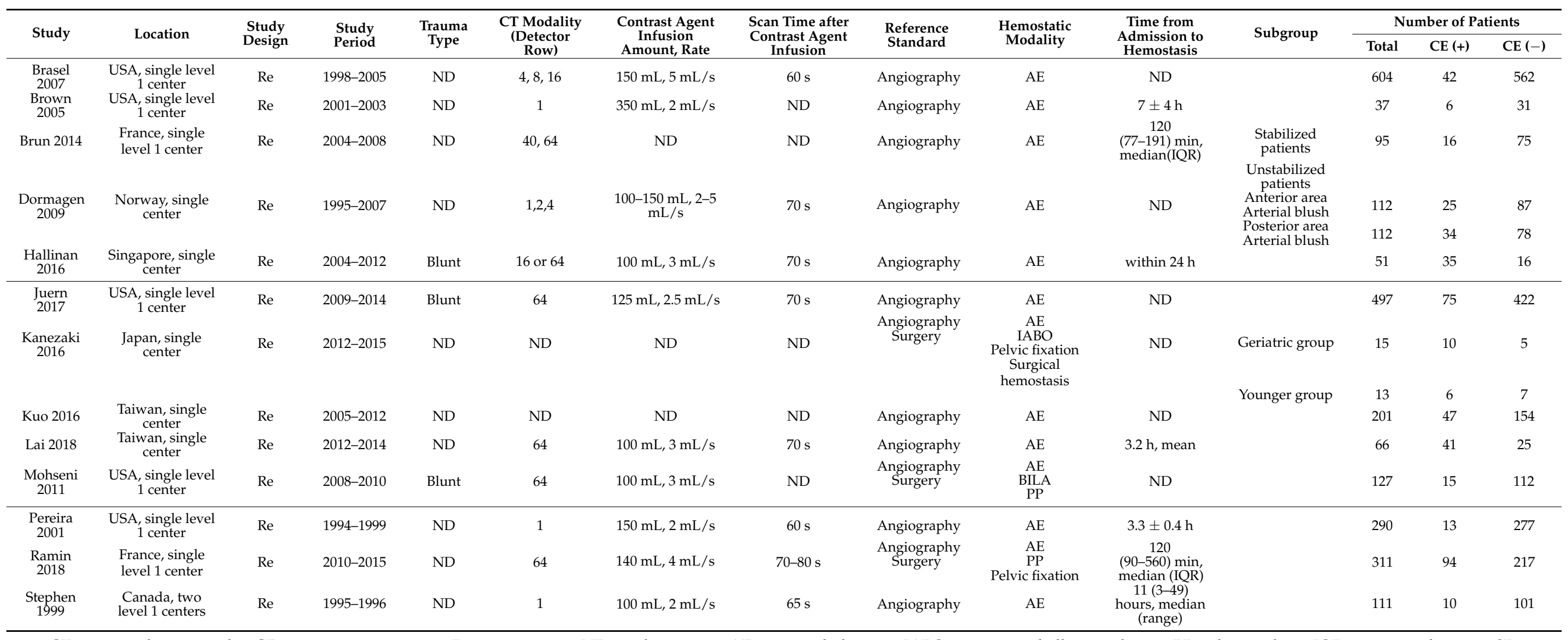

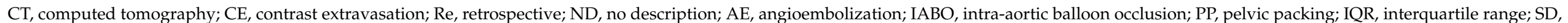
standard deviation; BILA, bilateral ligation of iliac arteries. 


\subsection{Clinical Characteristics of Patients and Contrast Extravasation on CT}

The estimated contrast extravasation positive rate of patients was 0.234 (95\% CI, $0.154-$ 0.334; heterogeneity test, $p<0.001$; Egger's test, $p=0.529 ; 13$ studies and 15 subsets) [10-22]. The estimated points of contrast clinical characteristics according to contrast extravasation on CT are summarized in Table 2 [10,14-16,19-21]. Age, injury severity score, and mortality rate were significantly higher in the positive-extravasation group.

Table 2. Estimate points of clinical characteristics according to contrast extravasation on computed tomography in patients with pelvic fracture.

\begin{tabular}{|c|c|c|c|c|c|}
\hline & $\begin{array}{l}\text { Number of } \\
\text { Subsets }\end{array}$ & Fixed Effect $(95 \%$ CI) & $\begin{array}{l}\text { Heterogeneity Test } \\
(p \text {-Value })\end{array}$ & $\begin{array}{l}\text { Random Effect } \\
\quad(95 \% \text { CI })\end{array}$ & $\begin{array}{c}\text { Egger's Test } \\
(p \text {-Value })\end{array}$ \\
\hline \multicolumn{6}{|c|}{ Age, mean } \\
\hline CE $(+)$ & 5 & $49.431(47.195,51.667)$ & 0.326 & $49.251(46.761,51.742)$ & 0.361 \\
\hline CE $(-)$ & 5 & $39.032(38.904,39.161)$ & $<0.001$ & $40.185(38.241,42.129)$ & 0.332 \\
\hline \multicolumn{6}{|c|}{ Sex, male rate } \\
\hline $\mathrm{CE}(+)$ & 5 & $0.623(0.559,0.683)$ & 0.074 & $0.615(0.511,0.710)$ & 0.660 \\
\hline CE $(-)$ & 5 & $0.601(0.577,0.625)$ & 0.279 & $0.603(0.575,0.631)$ & 0.110 \\
\hline \multicolumn{6}{|c|}{ ISS, mean } \\
\hline CE $(+)$ & 4 & $30.762(28.834,32.690)$ & $<0.001$ & $29.536(24.215,34.857)$ & 0.537 \\
\hline $\mathrm{CE}(-)$ & 4 & $18.210(18.117,18.304)$ & $<0.001$ & $19.184(17.707,20.660)$ & 0.413 \\
\hline \multicolumn{6}{|c|}{ Mortality, rate } \\
\hline CE $(+)$ & 7 & $0.163(0.125,0.208)$ & 0.682 & $0.163(0.125,0.208)$ & 0.078 \\
\hline CE $(-)$ & 5 & $0.057(0.045,0.072)$ & 0.015 & $0.048(0.029,0.078)$ & 0.046 \\
\hline
\end{tabular}

$\mathrm{CI}$, confidence interval; $\mathrm{CE}$, contrast extravasation; ISS, injury severity score.

\subsection{DTA Review}

The pooled sensitivity of CT was 0.786 (95\% CI, 0.574-0.909, I2 = 90\%), and the pooled specificity was 0.944 (95\% CI, 0.900-0.970, I2 = 88\%; Figure 2). The diagnostic odds ratio (DOR) of CT was 53.545 (95\% CI, 14.728-194.665) and the area under curve (AUC) on summary receiver operating characteristics (SROC) was 0.994 . There was no threshold effect by calculating Spearman's rank correlation coefficient $(r=-0.105, p=0.750)$.

\subsection{Sensitivity Analysis and Subgroup Analysis}

Two studies did not describe whether multi-detector CT was used or not (Table 1). For sensitivity analysis, studies with non-descriptive CT modalities or low-quality CT (1-4 detector row) were deleted (pooled sensitivity, 0.786 [95\% CI, 0.574-0.909] vs. 0.915 [95\% CI, 0.848-0.953]; pooled specificity, 0.944 [95\% CI, 0.900-0.970] vs. 0.906 [0.828-0.951]; Figure 3).

Subgroup analysis was performed according to the CT modality that is divided by the number of detector rows that affects the quality of the image (Figures 3 and 4 ). The pooled sensitivity of the 1-4 detector row group was low $(0.487$ [95\% CI, 0.215-0.767, I2 = 86\%]), while the pooled sensitivity of the 16-64 detector row group was high $(0.915$ [95\% CI, $0.848-0.953, \mathrm{I} 2=0 \%])$. The pooled specificity of the $1-4$ and $16-64$ detector row groups was high $(0.956$ [95\% CI, 0.876-0.985, I2 = 81\%] and 0.906 [95\% CI, 0.828-0.951, I2 = 72\%], respectively). The DOR was 19.582 (95\% CI, 1.909-200.872) in 1-4 detector row group and 76.178 (95\% CI, 29.261-198.320) in 16-64 detector row group, respectively. The AUC on SROC was 0.885 in 1-4 detector row group and 0.915 in 16-64 detector row group, respectively. There were no significant differences in the diagnostic accuracy of contrast extravasation on CT among the location of studies (Table 3). 


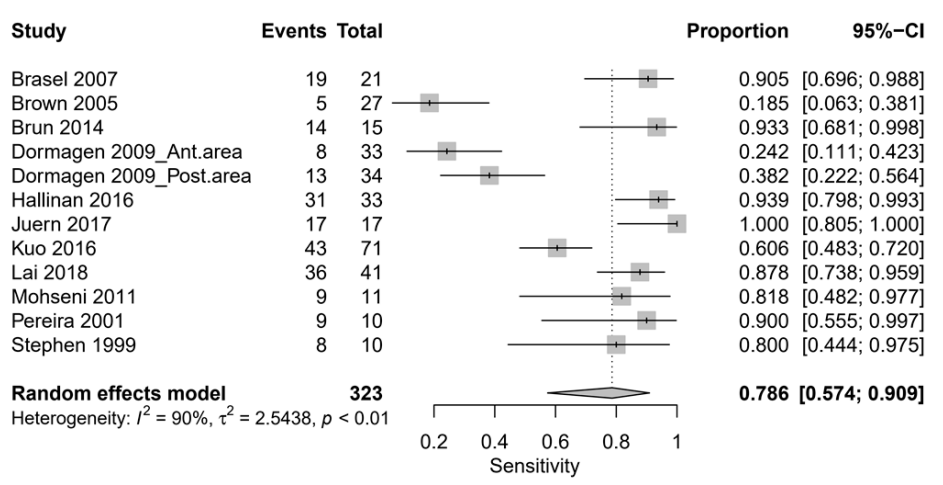

(A)

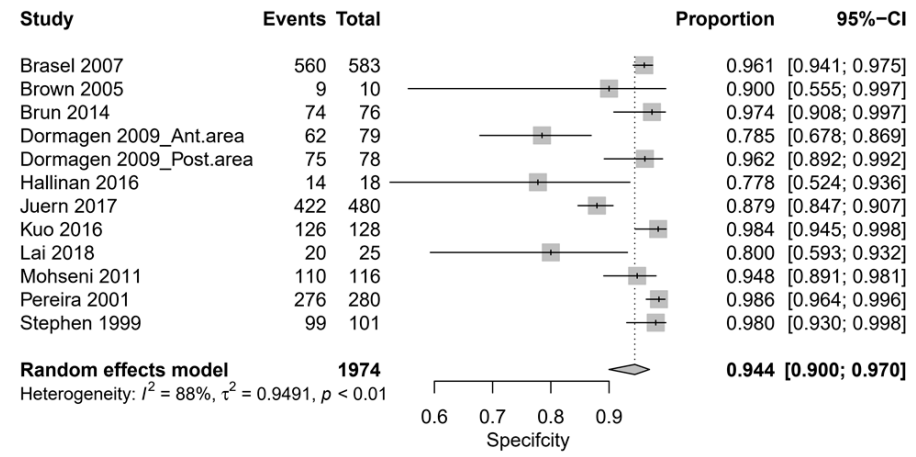

(B)

SROC curve (bivariate model) for Diagnostic Test Accuracy

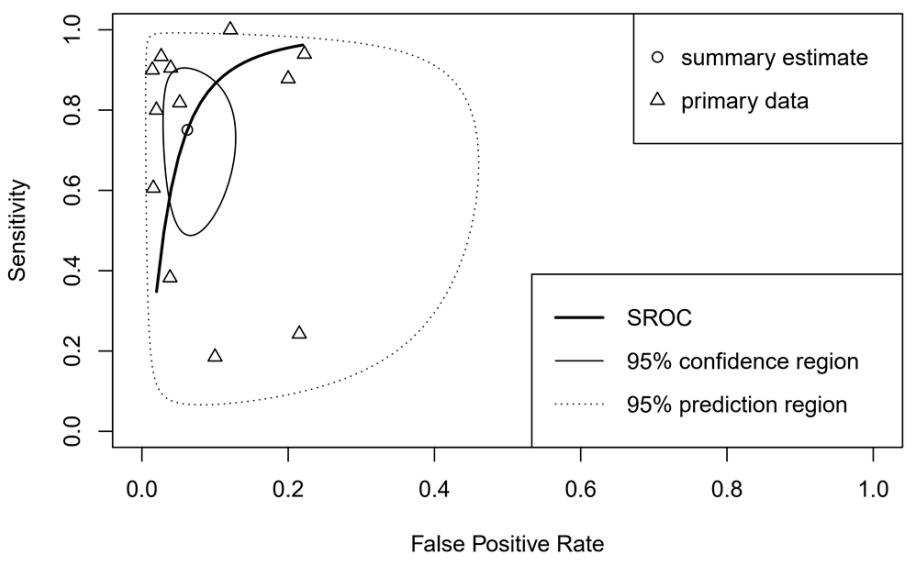

(C)

Figure 2. Forest plots for sensitivity (A) and specificity (B) and summary receiver operating characteristics (SROC) curve (C). 


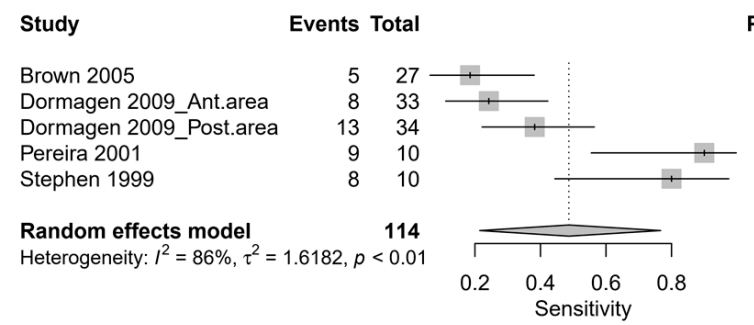

Proportion $\quad 95 \%-\mathrm{Cl}$

$0.185[0.063 ; 0.381]$

$0.242[0.111 ; 0.423]$

$0.382[0.222 ; 0.564]$

$0.900[0.555 ; 0.997]$

$0.800[0.444 ; 0.975]$

$0.487[0.215 ; 0.767]$

(A)

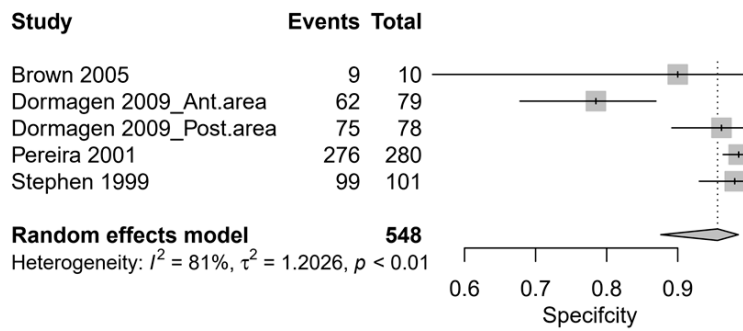

Proportion $\quad 95 \%-\mathrm{Cl}$

(B)

SROC curve (bivariate model) for Diagnostic Test Accuracy

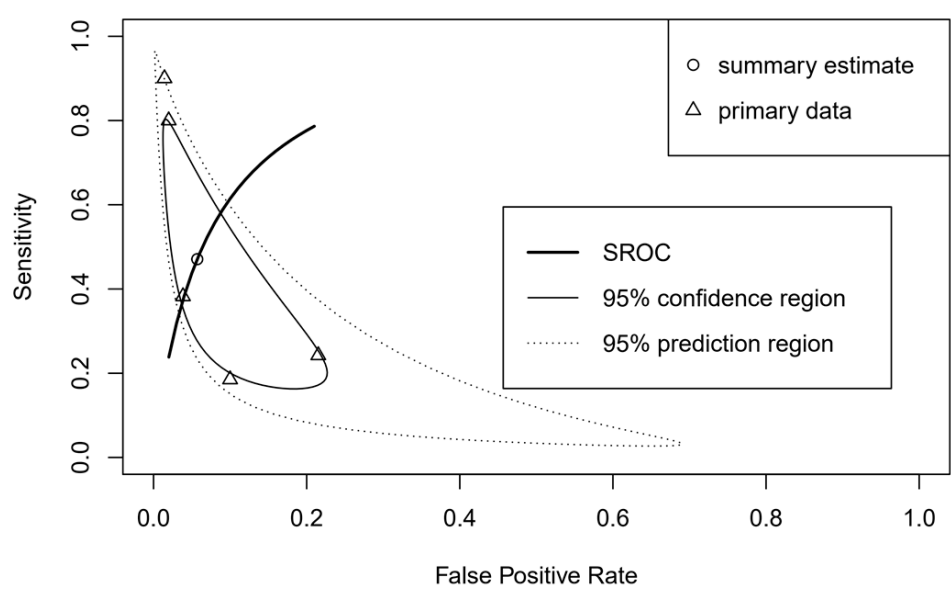

(C)

Figure 3. One to four detector row: forest plots for sensitivity (A), specificity (B), and SROC curve (C). 


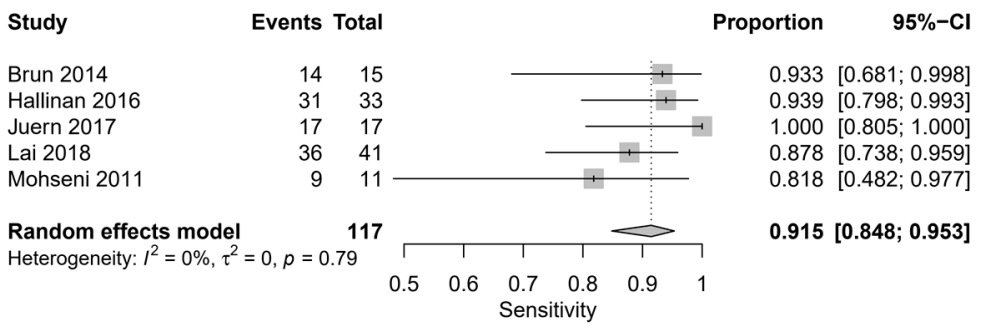

(A)

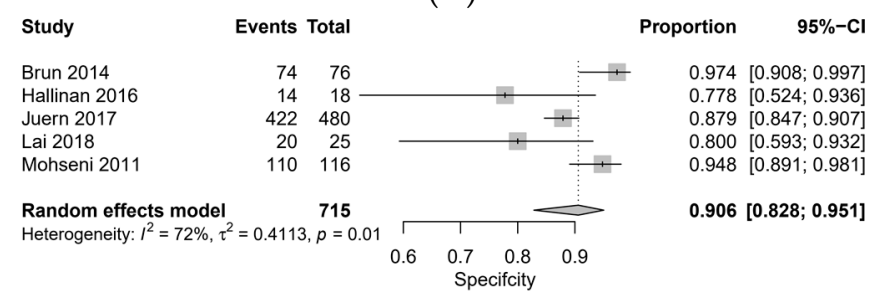

(B)

SROC curve (bivariate model) for Diagnostic Test Accuracy

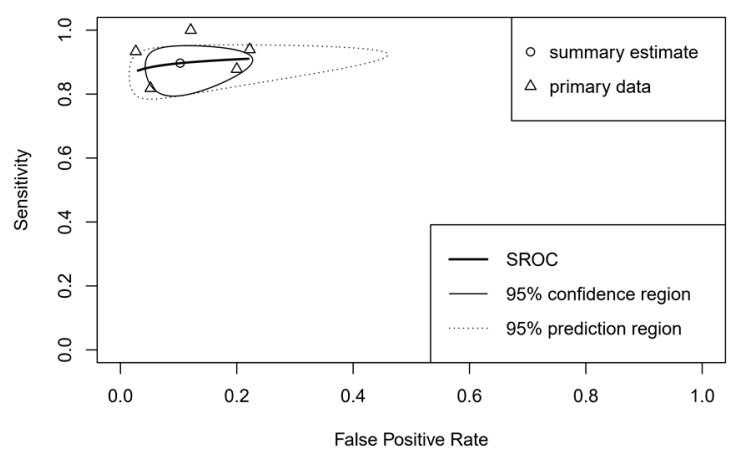

(C)

Figure 4. 16-64 detector row: forest plots for sensitivity (A), specificity (B), and SROC curve (C).

Table 3. Meta-regression of factors associated with diagnostic accuracy of contrast extravasation on computed tomography in patients with pelvic fracture.

\begin{tabular}{|c|c|c|c|c|c|c|c|}
\hline & Number & Fixed Effect & $\begin{array}{l}\text { Heterogeneity } \\
\text { Test }\end{array}$ & Random Effect & Egger's & \multirow{3}{*}{ Trim and Fill Test } & \multirow[b]{2}{*}{$\begin{array}{c}{ }^{*} \text { Meta- } \\
\text { Regression Test } \\
{[p \text {-Value }]}\end{array}$} \\
\hline & of & {$[95 \% \mathrm{CI}]$} & [ $p$-Value] & {$[95 \% \mathrm{CI}]$} & Test & & \\
\hline & Subsets & & & & [p-Value] & & \\
\hline Overall patients & 24 & $0.730(0.694,0.762]$ & $<0.001$ & $0.861(0.766,0.922]$ & 0.01 & $0.884(0.779,0.943]$ & \\
\hline America & 12 & $0.602(0.532,0.669]$ & $<0.001$ & $0.896(0.693,0.970]$ & $<0.001$ & $0.862(0.629,0.958]$ & \\
\hline Asia & 6 & $0.845(0.799,0.882]$ & 0.335 & $0.852(0.798,0.893]$ & 0.102 & $-0.00-10.0001$ & 0.948 \\
\hline Europe & 6 & $0.728(0.667,0.782]$ & $<0.001$ & $0.777(0.597,0.891)$ & 0.526 & - & 0.448 \\
\hline 1-4 detector row & 10 & $0.707(0.650,0.758]$ & $<0.001$ & $0.793(0.616,0.901]$ & 0.345 & - & \\
\hline America & 6 & $0.715(0.600,0.807]$ & $<0.001$ & $0.881(0.514,0.981]$ & 0.052 & - & \\
\hline Europe & 4 & $0.704(0.638,0.763]$ & $<0.001$ & $0.672(0.475,0.823]$ & 0.648 & - & 0.227 \\
\hline 16-64 detector row & 10 & $0.693(0.620,0.759]$ & $<0.001$ & $0.897(0.702,0.970]$ & $<0.001$ & $0.933(0.682,0.989]$ & \\
\hline America & 4 & $0 . .455(0.348,0.567]$ & $<0.001$ & $0.904(0.360,0.994]$ & 0.047 & $0.904(0.360,0.994]$ & \\
\hline Asia & 4 & $0.860(0.784,0.913)$ & 0.785 & $0.860(0.784,0.913]$ & 0.799 & - & 0.886 \\
\hline Europe & 2 & $0.942(0.834,0.982]$ & 0.061 & $0.954(0.674,0.995]$ & - & - & 0.563 \\
\hline CE positive patients & 12 & $0.566(0.499,0.632]$ & $<0.001$ & $0.723(0.533,0.856]$ & 0.003 & 0.655 (0.470. 0.802] & \\
\hline 1-4 detector row & 5 & $0.600(0.465,0.720]$ & 0.01 & $0.683(0.427,0.861]$ & 0.079 & $0.00010 .0 .002]$ & \\
\hline 16-64 detector row & 5 & $0.544(0.559,0.636]$ & $<0.001$ & $0.729(0.355,0.929]$ & 0.057 & - & \\
\hline CE negative patients & 12 & $0.814(0.714,0.845$ & $<0.001$ & $0.937(0.859,0.974]$ & 0.016 & $0.867(0.726,0.941]$ & \\
\hline 1-4 detector row & 5 & $0.736(0.674,0.789]$ & $<0.001$ & $0.867(0.634,0.961]$ & 0.343 & - & \\
\hline $16-64$ detector row & 5 & $0.934(0.882,0.964]$ & $<0.001$ & $0.968(0.855,0.994]$ & 0.052 & - & \\
\hline
\end{tabular}

$\mathrm{CI}$, confidence interval; ${ }^{*}$, compared to studies from America; $\mathrm{CE}$, contrast extravasation. 


\subsection{Quality Assessment}

The details of the quality assessment are described in Figure 5. In terms of test flow and timing, the risk of bias was unclear in eight studies (61.5\%). Only seven studies (53.8\%) showed the time from admission to hemostasis (angioembolization or surgery). Moreover, several studies had a long duration from admission to identify the bleeding via angiography or surgery $(7-24 \mathrm{~h})[11,14,22]$. Two studies had a significantly high risk of bias in terms of reference standards $[16,21]$. These studies include resuscitative endovascular balloon occlusion of the aorta (REBOA) and external pelvic fixation that are not amenable to directly identify the bleeding focus. Indeed, REBOA needs consecutive surgical or angiographic hemorrhagic control. We decided that these modalities are not suitable for the reference standard. Therefore, we did not calculate the sensitivity and specificity in these studies. In contrast to these two studies, one eligible study [19] included a bilateral internal iliac artery ligation through laparotomy that was amenable to directly identify the presence of bleeding.

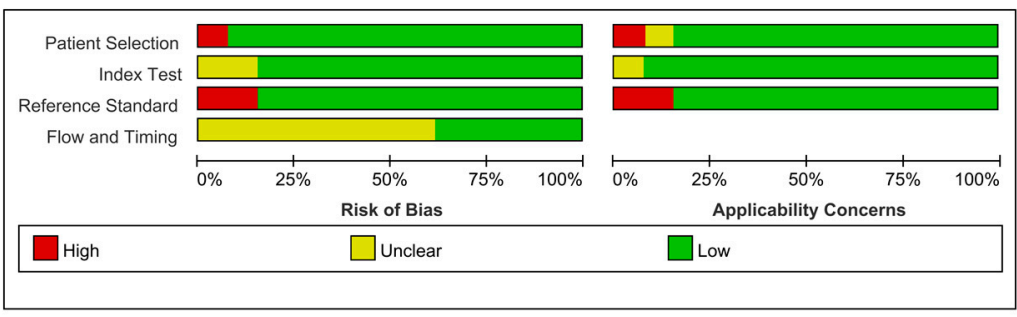

(A)

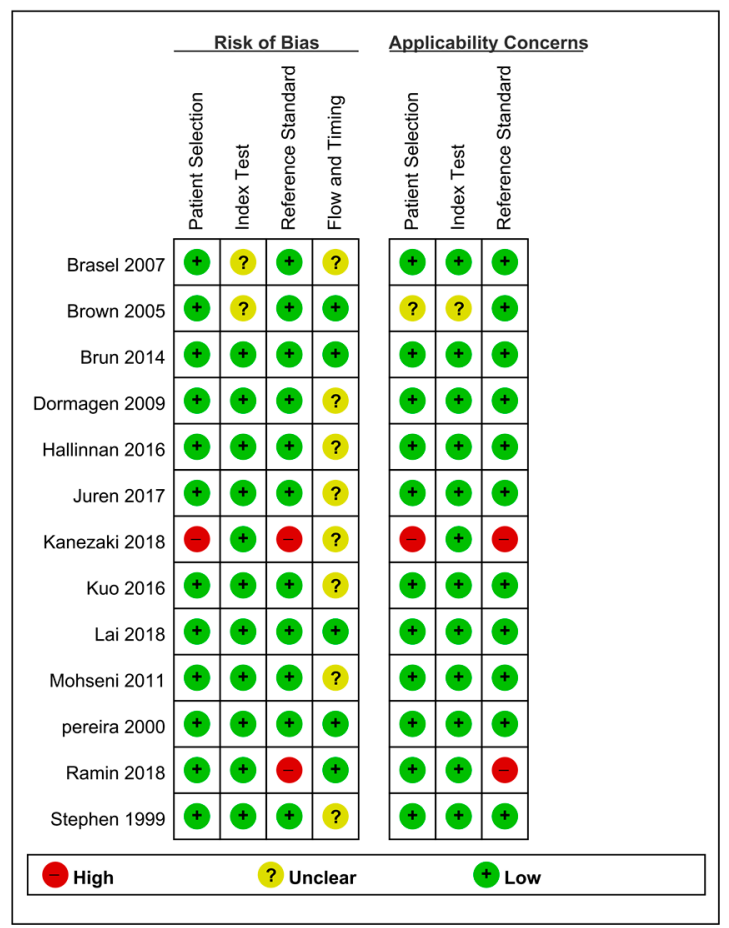

(B)

Figure 5. Risk of bias and applicability concerns graph (A) and summary (B): review authors' judgements about each domain presented as percentages across included studies.

\subsection{Publication Bias}

To assess publication bias, Begg's funnel plot and Egger's test were preferentially conducted. In the estimated mortality rate according to contrast extravasation, there was a significant publication bias ( $p=0.078$; Table 2). According to contrast extravasation, there were significant biases (overall, $p=0.010$; positive contrast extravasation, $p=0.003$; 
negative contrast extravasation, $p=0.016$; Table 3). To define the degree of publication bias, the fail-safe $\mathrm{N}$ and trim and fill tests were conducted as secondary assessments, and no significant publication bias was found (Table 3). In assessing other subgroups, no significant publication bias emerged.

\section{Discussion}

Our results suggest that contrast extravasation on the CT in pelvic trauma patients showed acceptable diagnostic accuracy, especially in multi-detector CT. In several early conducted studies, 1-4 detector row CT showed low sensitivity and limited diagnostic value. However, recent multi-detector row CT (16-64 detector row) showed sufficient sensitivity and specificity. In a recent meta-analysis, subgroup analysis showed pooled sensitivity and specificity of 94 and $89 \%$ for 64-detector row CT [8]. The present study demonstrated that even the 16-64 detector row CT showed sufficient sensitivity (91.5\%) and specificity (90.6\%). Furthermore, we noted that the pooled estimates of age, ISS, and mortality rate were higher in contrast extravasation group. Whereas, we defined more strict criteria regarding the reference test that comprised angiography and surgical diagnosis (direct inspection by a surgeon), the previous meta-analysis [8] used ambiguous reference tests such as external fixation or preperitoneal pelvic packing that are not able to identify the real arterial hemorrhage directly. We also defined more strict criteria regarding positive angiography, whereas the previous meta-analysis [8] comprised decision of embolization. This contributed to a smaller number of eligible studies in our analysis. A rigorous and explicit definition of reference standard should be needed to reduce the heterogeneity and the risk of bias in diagnostic test accuracy review [25]. Nevertheless, there was heterogeneity and substantial risk of bias in terms of timing of index test and reference standard in the present study.

In the present study, we focused on extravasation on CT that indicates bleeding from the injured vessel. In contrast to our results, in a retrospective review including 162 pelvic ring fracture patients, pelvic blushes with stable vital signs were successfully managed without surgical or radiological hemorrhagic control [26]. Whereas the role of CT is limited in hemodynamically unstable patients [27], the pelvic angiography/angioembolization, in patients with arterial contrast extravasation on CT, may have a benefit regardless of hemodynamic status [28] Clinically, false-negatives for pelvic hemorrhage are more dangerous than false-positives, because a failure to recognize the underlying bleeding can lead to catastrophe. The false-negative rates of the present analysis were $51.3 \%$ in 1-4 detector row and $8.5 \%$ in 16-64 detector row, respectively. In a recent retrospective review using modern 64-detector row CT scanners [15], 100\% negative predictive value was reported, whereas another previous study [17] reported 28 positive angiography patients among 154 negative contrast blushes on CT (18.2\%). However, the detector row type of the CT scanner was not described in that study. Currently, the absence of contrast blush cannot exclude active pelvic bleeding [28].

In our analysis, we found that multi-detector CT has sufficient sensitivity and specificity for detecting severe hemorrhage. In a recent meta-analysis, no serious pelvic injuries were found when physical examination findings were normal [29]. A portable pelvic radiograph is not an effective diagnostic tool to detect pelvic fractures, because it often failed to detect sacral and iliac fractures [30]. Focused abdominal sonography for trauma (FAST) is a useful option for early detection of intraperitoneal fluid (hemorrhage), but it is limited to the detection of retroperitoneal hemorrhages $[27,31]$. The previously reported diagnostic accuracy of FAST for hemorrhagic pelvic fracture was poor $(26 \%$ sensitivity, $96 \%$ specificity, $85 \%$ positive predictive value, and $63 \%$ negative predictive value, respectively) [32]. Nevertheless, many clinicians still prefer FAST to CT in unstable patients due to the limitation of CT [33]. A recent randomized trial [34] regarding whole-body CT (WBCT) for severe trauma patients did not reveal a significant difference in terms of inhospital mortality, whereas a meta-analysis [35] revealed a favorable outcome (pooled odds ratio in WBCT group for $24 \mathrm{~h}$ morality, 0.72 [95\% CI, 0.66-0.79]). Therefore, CT remains a 
controversial initial diagnostic tool in unstable patients. The recent management algorithm of pelvic fracture depends on hemodynamic instability [27]. Pelvic injuries range from low-energy simple fractures to high-energy unstable patterns that can lead to severe lethal hemorrhages. Thus, emergent resuscitation should be a priority over CT scanning in unstable patients. Recently, various treatment modalities, including hemostatic resuscitation guided by viscoelastic testing, REBOA, preperitoneal pelvic packing, and external fixation, are performed without CT scanning, according to hemodynamic instability, although the exact role of REBOA is not determined yet [2,27]. For CT scanning, the patient should be transferred to the CT room from the resuscitation or emergency room, where it will take tens of minutes to complete the examination. Since this is a time-consuming procedure and the patient's instability can worsen, ultrasonography or portable plain pelvic radiography is preferred over CT scanning [36].

The present study has several limitations. First, all eligible studies were retrospective, thus selection bias could not be avoided. Second, the present study has limitations concerning the heterogeneity of reported data. Although the statistical heterogeneity was substantially high in all eligible studies, the studies using 16-64 detector row CT showed low heterogeneity after subgroup analysis as well as sufficient summary estimates of sensitivity. There was substantial heterogeneity across the studies regarding the duration from admission to reference standard. The description of the timing of CT scanning was absent in all studies. Thus, the appropriate interval between index test and reference standard is unclear. This suggests the need for future application and investigation of the hybrid-ER system. Third, the quality of CT modalities varied across the studies. However, by performing subgroup analysis, we revealed that modern multi-detector CT has acceptable diagnostic accuracy. Fourth, the publication-bias-adjusted pooled estimates after using the trim-and-fill method were not significantly different. However, the small number of studies in the present meta-analysis could provide little precision [37]. Finally, we could not separate the datasets according to the hemodynamic status. Thus, a further study addressing the diagnostic value of CT alone, regardless of vital signs, is warranted.

\section{Conclusions}

Our meta-analysis demonstrated that modern multi-detector CT, with 16 or more detector rows, has acceptable high sensitivity and specificity, whereas 1-4 detector row CT has limitations in diagnosis. We found that even the $\mathrm{CT}$ with 16 detector rows has sufficient accuracy compared to the previous meta-analysis [8]. Extravasation on CT indicates severe hemorrhage in pelvic fracture patients.

Author Contributions: Conceptualization, S.N.M., J.-S.P., and W.S.K.; methodology, S.N.M., J.-S.P., and W.S.K.; software, J.-S.P. and W.S.K.; validation, S.N.M., J.-S.P., and W.S.K.; formal analysis, J.-S.P. and W.S.K.; investigation, S.N.M., J.-S.P., and W.S.K.; resources, S.N.M., J.-S.P., and W.S.K.; data curation, J.-S.P. and W.S.K.; writing-original draft preparation, S.N.M., J.-S.P., and W.S.K.; writing-review and editing, S.N.M., J.-S.P., and W.S.K.; visualization, S.N.M., J.-S.P., and W.S.K.; supervision, S.N.M., J.-S.P., and W.S.K. All authors have read and agreed to the published version of the manuscript.

Funding: This research received no external funding.

Institutional Review Board Statement: The study was conducted according to the guidelines of the Declaration of Helsinki, and approved by the Institutional Review Board of Wonkwang University Hospital (WKUH 2019-10-035).

Informed Consent Statement: Patient consent was waived due to retrospective nature of the study.

Data Availability Statement: Data is contained within the article.

Acknowledgments: This work was supported by the Korea Medical Device Development Fund grant funded by the Korea government (the Ministry of Science and ICT, the Ministry of Trade, Industry and Energy, the Ministry of Health and Welfare, Republic of Korea, the Ministry of Food and Drug Safety) (Project Number: 202012B04). 
Conflicts of Interest: The authors declare no conflict of interest.

\section{References}

1. Jang, J.Y.; Shim, H.; Jung, P.Y.; Kim, S.; Bae, K.S. Preperitoneal pelvic packing in patients with hemodynamic instability due to severe pelvic fracture: Early experience in a Korean trauma center. Scand. J. Trauma Resusc. Emerg. Med. 2016, 24, 1-7. [CrossRef] [PubMed]

2. $\quad$ Burlew, C.C.; Moore, E.E.; Stahel, P.F.; Geddes, A.E.; Wagenaar, A.E.; Pieracci, F.M.; Fox, C.J.; Campion, E.M.; Johnson, J.L.; Mauffrey, C. Preperitoneal pelvic packing reduces mortality in patients with life-threatening hemorrhage due to unstable pelvic fractures. J. Trauma Acute Care Surg. 2017, 82, 233. [CrossRef] [PubMed]

3. Costantini, T.W.; Coimbra, R.; Holcomb, J.B.; Podbielski, J.M.; Catalano, R.; Blackburn, A.; Scalea, T.M.; Stein, D.M.; Williams, L.; Conflitti, J. Current management of hemorrhage from severe pelvic fractures: Results of an American Association for the Surgery of Trauma multi-institutional trial. J. Trauma Acute Care Surg. 2016, 80, 717-725. [CrossRef] [PubMed]

4. Pommerening, M.J.; Goodman, M.D.; Holcomb, J.B.; Wade, C.E.; Fox, E.E.; del Junco, D.J.; Brasel, K.J.; Bulger, E.M.; Cohen, M.J.; Alarcon, L.H. Clinical gestalt and the prediction of massive transfusion after trauma. Injury 2015, 46, 807-813. [CrossRef] [PubMed]

5. Tran, A.; Matar, M.; Lampron, J.; Steyerberg, E.; Taljaard, M.; Vaillancourt, C. Early identification of patients requiring massive transfusion, embolization or hemostatic surgery for traumatic hemorrhage: A systematic review and meta-analysis. J. Trauma Acute Care Surg. 2018, 84, 505-516. [CrossRef] [PubMed]

6. Kinoshita, T.; Yamakawa, K.; Matsuda, H.; Yoshikawa, Y.; Wada, D.; Hamasaki, T.; Ono, K.; Nakamori, Y.; Fujimi, S. The survival benefit of a novel trauma workflow that includes immediate whole-body computed tomography, surgery, and interventional radiology, all in one trauma resuscitation room: A retrospective historical control study. Ann. Surg. 2019, 269, 370. [CrossRef] [PubMed]

7. Wada, D.; Nakamori, Y.; Yamakawa, K.; Fujimi, S. First clinical experience with IVR-CT system in the emergency room: Positive impact on trauma workflow. Scand. J. Trauma Resusc. Emerg. Med. 2012, 20, 52. [CrossRef]

8. Dreizin, D.; Liang, Y.; Dent, J.; Akhter, N.; Mascarenhas, D.; Scalea, T.M. Diagnostic value of CT contrast extravasation for major arterial injury after pelvic fracture: A meta-analysis. Am. J. Emerg. Med. 2019, 38, 2335-2342. [CrossRef]

9. McInnes, M.D.; Moher, D.; Thombs, B.D.; McGrath, T.A.; Bossuyt, P.M.; Clifford, T.; Cohen, J.F.; Deeks, J.J.; Gatsonis, C.; Hooft, L. Preferred reporting items for a systematic review and meta-analysis of diagnostic test accuracy studies: The PRISMA-DTA statement. JAMA 2018, 319, 388-396. [CrossRef]

10. Brasel, K.J.; Pham, K.; Yang, H.; Christensen, R.; Weigelt, J.A. Significance of contrast extravasation in patients with pelvic fracture. J. Trauma Acute Care Surg. 2007, 62, 1149-1152. [CrossRef]

11. Brown, C.V.; Kasotakis, G.; Wilcox, A.; Rhee, P.; Salim, A.; Demetriades, D. Does pelvic hematoma on admission computed tomography predict active bleeding at angiography for pelvic fracture? Am. Surg. 2005, 71, 759-762. [CrossRef] [PubMed]

12. Brun, J.; Guillot, S.; Bouzat, P.; Broux, C.; Thony, F.; Genty, C.; Heylbroeck, C.; Albaladejo, P.; Arvieux, C.; Tonetti, J.; et al. Detecting active pelvic arterial haemorrhage on admission following serious pelvic fracture in multiple trauma patients. Injury 2014, 45, 101-106. [CrossRef] [PubMed]

13. Dormagen, J.B.; Tötterman, A.; Røise, O.; Sandvik, L.; Kløw, N.E. Efficacy of plain radiography and computer tomography in localizing the site of pelvic arterial bleeding in trauma patients. Acta Radiol. 2010, 51, 107-116. [CrossRef] [PubMed]

14. Hallinan, J.T.P.D.; Tan, C.H.; Pua, U. The role of multidetector computed tomography versus digital subtraction angiography in triaging care and management in abdominopelvic trauma. Singap. Med. J. 2016, 57, 497. [CrossRef] [PubMed]

15. Juern, J.S.; Milia, D.; Codner, P.; Beckman, M.; Somberg, L.; Webb, T.; Weigelt, J.A. Clinical significance of computed tomography contrast extravasation in blunt trauma patients with a pelvic fracture. J. Trauma Acute Care Surg. 2017, 82, 138-140. [CrossRef] [PubMed]

16. Kanezaki, S.; Miyazaki, M.; Notani, N.; Tsumura, H. Clinical presentation of geriatric polytrauma patients with severe pelvic fractures: Comparison with younger adult patients. Eur. J. Orthop. Surg. Traumatol. 2016, 26, 885-890. [CrossRef] [PubMed]

17. Kuo, L.-W.; Yang, S.-J.; Fu, C.-Y.; Liao, C.-H.; Wang, S.-Y.; Wu, S.-C. Relative hypotension increases the probability of the need for angioembolisation in pelvic fracture patients without contrast extravasation on computed tomography scan. Injury 2016, 47 , 37-42.

18. Lai, Y.-C.; Wu, C.-H.; Chen, H.-W.; Wang, L.-J.; Wong, Y.-C. Predictors of active arterial hemorrhage on angiography in pelvic fracture patients. Jpn. J. Radiol. 2018, 36, 223-230. [CrossRef]

19. Mohseni, S.; Talving, P.; Kobayashi, L.; Lam, L.; Inaba, K.; Branco, B.C.; Oliver, M.; Demetriades, D. The diagnostic accuracy of 64-slice computed tomography in detecting clinically significant arterial bleeding after pelvic fractures. Am. Surg. 2011, 77, 1176-1182. [CrossRef]

20. Pereira, S.J.; O’Brien, D.P.; Luchette, F.A.; Choe, K.A.; Lim, E.; Davis, K., Jr.; Hurst, J.M.; Johannigman, J.A.; Frame, S.B. Dynamic helical computed tomography scan accurately detects hemorrhage in patients with pelvic fracture. Surgery 2000, 128, 678-685.

21. Ramin, S.; Hermida, M.; Millet, I.; Murez, T.; Monnin, V.; Hamoui, M.; Capdevila, X.; Charbit, J. Limits of intravascular contrast extravasation on computed tomography scan to define the need for pelvic angioembolization in pelvic blunt trauma: A specific assessment on the risk of false positives. J. Trauma Acute Care Surg. 2018, 85, 527-535. [CrossRef] [PubMed] 
22. Stephen, D.J.; Kreder, H.J.; Day, A.C.; McKee, M.D.; Schemitsch, E.H.; ElMaraghy, A.; Hamilton, P.; McLellan, B. Early detection of arterial bleeding in acute pelvic trauma. J. Trauma Acute Care Surg. 1999, 47, 638. [CrossRef] [PubMed]

23. Whiting, P.; Rutjes, A.W.; Reitsma, J.B.; Bossuyt, P.M.; Kleijnen, J. The development of QUADAS: A tool for the quality assessment of studies of diagnostic accuracy included in systematic reviews. BMC Med. Res. Methodol. 2003, 3, 25. [CrossRef] [PubMed]

24. Borenstein, M.; Hedges, L.V.; Higgins, J.P.; Rothstein, H.R. Introduction to Meta-Analysis; John Wiley \& Sons, Ltd.: Hoboken, NJ, USA, 2009.

25. Macaskill, P.; Gatsonis, C.; Deeks, J.; Harbord, R.; Takwoingi, Y. Cochrane Handbook for Systematic Reviews of Diagnostic Test Accuracy; Version 0.9.0; The Cochrane Collaboration: London, UK, 2010.

26. Verbeek, D.O.; Zijlstra, I.A.; van der Leij, C.; Ponsen, K.J.; van Delden, O.M.; Goslings, J.C. Management of pelvic ring fracture patients with a pelvic "blush" on early computed tomography. J. Trauma Acute Care Surg. 2014, 76, 374-379. [CrossRef]

27. Tran, T.L.N.; Brasel, K.J.; Karmy-Jones, R.; Rowell, S.; Schreiber, M.A.; Shatz, D.V.; Albrecht, R.M.; Cohen, M.J.; DeMoya, M.A.; Biffl, W.L. Western Trauma Association Critical Decisions in Trauma: Management of pelvic fracture with hemodynamic instability-2016 updates. J. Trauma Acute Care Surg. 2016, 81, 1171-1174. [CrossRef]

28. Coccolini, F.; Stahel, P.F.; Montori, G.; Biffl, W.; Horer, T.M.; Catena, F.; Kluger, Y.; Moore, E.E.; Peitzman, A.B.; Ivatury, R. Pelvic trauma: WSES classification and guidelines. World J. Emerg. Surg. 2017, 12, 1-18. [CrossRef]

29. van Trigt, J.; Schep, N.W.; Peters, R.W.; Goslings, J.C.; Schepers, T.; Halm, J.A. Routine pelvic X-rays in asymptomatic hemodynamically stable blunt trauma patients: A meta-analysis. Injury 2018, 49, 2024-2031. [CrossRef]

30. Stewart, B.G.; Rhea, J.T.; Sheridan, R.L.; Novelline, R.A. Is the screening portable pelvis film clinically useful in multiple trauma patients who will be examined by abdominopelvic CT? Experience with 397 patients. Emerg. Radiol. 2002, 9, 266-271. [CrossRef]

31. Richards, J.R.; McGahan, J.P. Focused assessment with sonography in trauma (FAST) in 2017: What radiologists can learn. Radiology 2017, 283, 30-48. [CrossRef]

32. Friese, R.S.; Malekzadeh, S.; Shafi, S.; Gentilello, L.M.; Starr, A. Abdominal ultrasound is an unreliable modality for the detection of hemoperitoneum in patients with pelvic fracture. J. Trauma Acute Care Surg. 2007, 63, 97-102. [CrossRef]

33. Grünherz, L.; Jensen, K.O.; Neuhaus, V.; Mica, L.; Werner, C.M.; Ciritsis, B.; Michelitsch, C.; Osterhoff, G.; Simmen, H.-P.; Sprengel, K. Early computed tomography or focused assessment with sonography in abdominal trauma: What are the leading opinions? Eur. J. Trauma Emerg. Surg. 2018, 44, 3-8. [CrossRef] [PubMed]

34. Sierink, J.C.; Treskes, K.; Edwards, M.J.; Beuker, B.J.; den Hartog, D.; Hohmann, J.; Dijkgraaf, M.G.; Luitse, J.S.; Beenen, L.F.; Hollmann, M.W. Immediate total-body CT scanning versus conventional imaging and selective CT scanning in patients with severe trauma (REACT-2): A randomised controlled trial. Lancet 2016, 388, 673-683. [CrossRef]

35. Chidambaram, S.; Goh, E.L.; Khan, M.A. A meta-analysis of the efficacy of whole-body computed tomography imaging in the management of trauma and injury. Injury 2017, 48, 1784-1793. [CrossRef] [PubMed]

36. The ATLS Subcommittee; American College of Surgeons' Committee on Trauma; The International ATLS Working Group. Advanced trauma life support $\left(\right.$ ATLS $\left.^{\circledR}\right)$ : The ninth edition. J. Trauma Acute Care Surg. 2013, 74, 1363-1366.

37. Lau, J.; Ioannidis, J.P.; Terrin, N.; Schmid, C.H.; Olkin, I. The case of the misleading funnel plot. BMJ 2006, 333, 597-600. [CrossRef] [PubMed] 